\title{
Kronos: exploring the depths of Saturn with probes and remote sensing through an international mission
}

\author{
B. Marty • T. Guillot • A. Coustenis - the Kronos consortium • \\ N. Achilleos · Y. Alibert - S. Asmar - D. Atkinson - S. Atreya • \\ G. Babasides $\cdot$ K. Baines $\cdot$ T. Balint $\cdot$ D. Banfield $\cdot$ S. Barber • \\ B. Bézard - G. L. Bjoraker · M. Blanc - S. Bolton - N. Chanover • \\ S. Charnoz • E. Chassefière · J. E. Colwell · E. Deangelis • \\ M. Dougherty • P. Drossart • F. M. Flasar • T. Fouchet • \\ R. Frampton - I. Franchi • D. Gautier • L. Gurvits • R. Hueso • \\ B. Kazeminejad - T. Krimigis - A. Jambon • G. Jones • Y. Langevin • \\ M. Leese · E. Lellouch • J. Lunine - A. Milillo • P. Mahaffy • \\ B. Mauk • A. Morse - M. Moreira - X. Moussas - C. Murray • \\ I. Mueller-Wodarg • T. C. Owen • S. Pogrebenko • R. Prangé • \\ P. Read - A. Sanchez-Lavega $\cdot$ P. Sarda - D. Stam • G. Tinetti • \\ P. Zarka · J. Zarnecki · J. Schmidt · H. Salo
}

Published online: 5 August 2008

(C) Springer Science + Business Media B.V. 2008

\section{Erratum to: Exp Astron \\ DOI 10.1007/s10686-008-9094-9}

In the originally published article, the names of co-authors Juergen Schmidt and Heikki Salo were omitted erroneously. The complete authorship is shown here.

The online version of the original article can be found under doi:10.1007/s10686-008-9094-9.

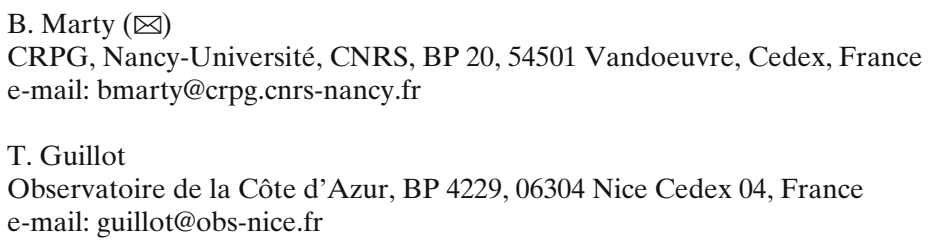

A. Coustenis · B. Bézard · P. Drossart · T. Fouchet · D. Gautier ·

E. Lellouch · R. Prangé · P. Zarka

Laboratoire d'Etudes Spatiales et d'Instrumentation en Astrophysique (LESIA),

Observatoire de Paris-Meudon, 5, place Jules Janssen, 92195 Meudon Cedex, France

A. Coustenis
e-mail: Athena.Coustenis@obspm.fr 
N. Achilleos

Atmospheric Physics Laboratory, Department of Physics and Astronomy, University College London, Gower Street, London WC1E 6BT, UK

Y. Alibert

Inst Phys, University of Bern, CH-3012 Bern, Switzerland

S. Asmar

Jet Propulsion Laboratory, Pasadena, CA 91109, USA

D. Atkinson

Department of Electrical and Computer Engineering, University of Idaho, Moscow, ID 83844-1023, USA

S. Atreya

Department of Atmosphere Ocean and Space Science,

University of Michigan, Ann Arbor, MI 48109, USA

G. Babasides $\cdot$ X. Moussas

Space Group, Laboratory of Astrophysics, Faculty of Physics,

National and Kapodistrian University of Athens,

Panepistimiopolis, 15783 Zographos, Athens, Greece

K. Baines • T. Balint

Jet Propulsion Laboratory, 4800 Oak Grove Blvd, Paadena, CA 91109-8099, USA

D. Banfield

Department of Astronomy, Cornell University, Ithaca, NY 14853, USA

S. Barber • I. Franchi • M. Leese • A. Morse • J. Zarnecki

Open University, Walton Hall, Milton Keynes MK7 6AA, UK

G. L. Bjoraker • F. M. Flasar • P. Mahaffy

NASA, Goddard Space Flight Ctr Code 693, Greenbelt, MD 20771, USA

M. Blanc

Centre d'Etudes Spatiales des Rayonnements (CESR), Toulouse, France

S. Bolton

Southwest Research Institute, San Antonio, TX, USA

N. Chanover

New Mexico State University, Las Cruces, NM 88003, USA

Springer 


\section{S. Charnoz}

AIM, Université Paris 7/CEA/CNRS, 91191 Gif sur Yvette, France

E. Chassefière

Service d'Aéronomie du CNRS/IPSL, 91371 Verrières-le-Buisson, France

J. E. Colwell

Department of Physics, University Cent Florida, Orlando, FL 32816, USA

E. Deangelis • A. Milillo

NAF/Instituto di Fisica dello Spazio Interplanetario,

via del Fosso del Cavaliere 100, 00133, Rome, Italy

\section{Dougherty}

Imperial College London, South Kensington Campus, London SW7 2AZ, UK

\section{Mueller-Wodarg}

Imperial College Sci Technol and Med, Space and Atmosphere Phs grp,

University of London, London SW7 2BW, UK

R. Frampton

Boeing NASA Systems, MC H012-C349, 5301 Bolsa Ave,

Huntington Beach, CA 92647-2099, USA

L. Gurvits $\cdot$ S. Pogrebenko

Joint Institute for VLBI in Europe, P.O. Box 2, 7990 AA Dwingeloo, The Netherlands

R. Hueso $\cdot$ A. Sanchez-Lavega

Departamento de Fisica Aplicada I, E.T.S. Ingenieros,

Universidad del Pais Vasco, Alameda Urquijo s/n, 48013 Bilbao, Spain

B. Kazeminejad

Deutsches Zentrum für Luft-und Raumfahrt (DLR),

German Space Operations Center (GSOC), 82234 Wessling, Germany

T. Krimigis • B. Mauk

Appl Phys Lab, Johns Hopkins University, Laurel, MD 20723, USA

\section{A. Jambon}

MAGIE UMR 7047, Université Pierre et Marie Curie,

4 place Jussieu, 75252 Paris Cedex 05, France 


\section{G. Jones}

Max Plank Inst. Gravitat Phys, Albert Einstein Inst, Katlenburg-Lindau, Germany

Y. Langevin

Institut d'Astrophysique Spatiale Bat. 121, 91405 Orsay Campus, France

J. Lunine

Department of Planetary Science, University of Arizona, Tucson, AZ 85721, USA

M. Moreira

Laboratoire de Géochimie et Cosmochimie (UMR 7579 CNRS),

Institut de Physique du Globe de Paris, Université Paris, 7,

4 place Jussieu, 75252 Paris cedex 05, France

C. Murray

Queen Mary \& Westfield College, University of London, London E1 4NS, UK

T. C. Owen

Institute of Astronomy, University of Hawaii, Honolulu, HI 96822, USA

P. Read

Clarendon Laboratory, University of Oxford, Oxford OX1 3PU, UK

P. Sarda

Groupe géochimie des Gaz Rares, Département des Sciences de la Terre,

Université Paris Sud, UMR CNRS 8148 (IDES), 81405 Orsay Cedex, France

D. Stam

Astronomical Institute "Anton Pannekoek" Kruislaan 403, 1098 SJ Amsterdam, The Netherlands

G. Tinetti

Institut d'Astrophysique de Paris, CNRS, Université Pierre et Marie Curie, 75014 Paris, France

Present address:

P. Sarda

Laboratoire de Sciences de la Terre, Ecole Normale Supérieure de Lyon cedex 07, France

J. Schmidt

Universitaet Potsdam, Institut fuer Physik und Astronomie,

Karl-Liebknecht-Str. 24/25, D-14476 Potsdam-Golm, Germany

H. Salo

Department of Physical Sciences, Astronomy Division,

University of Oulu, FI-90014 Oulu, Finland

e-mail: heikki.salo@oulu.fi 